\title{
On Optimal GFSR Pseudorandom Number Generators
}

\section{By Shu Tezuka}

\begin{abstract}
It is shown that in $t(\geq 4)$ dimensions no optimal GFSR generators exist.
\end{abstract}
1. Introduction. The binary representation of $p$-bit GFSR pseudorandom numbers is defined [1], [2] as follows, for given $j_{1}, j_{2}, \ldots, j_{p}$,

$$
X i=a_{j_{1}+i-1} a_{j_{2}+i-1} \cdots a_{j_{p}+i-1} \text { for } i=1,2,3, \ldots,
$$

where $\{X i\}$ is a sequence of $p$-bit integers and $\left\{a_{i}\right\}$ is an $M$-sequence with period length $2^{p}-1$ whose characteristic polynomial is

$$
f(D)=1+c_{1} D+c_{2} D^{2}+\cdots+c_{p-2} D^{p-2}+c_{p-1} D^{p-1}+D^{p}(\bmod 2) .
$$

Here, the initial values $\left(a_{1}, a_{2}, \ldots, a_{p}\right) \neq(0,0, \ldots, 0)$.

The GFSR sequences can be expressed by using the companion matrix of $M$ sequences. Denote the companion matrix by $C$,

$$
C=\left[\begin{array}{ccccrr}
0 & 1 & 0 & \cdots & 0 & 0 \\
0 & 0 & 1 & \cdots & 0 & 0 \\
0 & 0 & 0 & \cdots & 0 & 0 \\
& & \ldots & \cdots & \ldots & \\
& & \ldots & \cdots & \ldots & \\
0 & 0 & 0 & \cdots & 0 & 0 \\
0 & 0 & 0 & \cdots & 1 & 0 \\
0 & 0 & 0 & \cdots & 0 & 1 \\
1 & c_{p-1} & c_{p-2} & \cdots & c_{2} & c_{1}
\end{array}\right] .
$$

Let* $\alpha=\left(a_{1}, a_{2}, \ldots, a_{p}\right)^{t}, \beta=\left(a_{j_{1}}, a_{j_{2}}, \ldots, a_{j_{p}}\right)^{t}$. Then there exists a matrix $G$ such that $\beta=G \alpha$. Hence, a $p$-bit GFSR sequence can be expressed as follows,

$$
G \alpha, G C \alpha, G C^{2} \alpha, \ldots, G C^{i} \alpha, \ldots
$$

Assume that $G$ is nonsingular. Then the above sequence is

$$
\beta, T \beta, T^{2} \beta, \ldots, T^{i} \beta, \ldots,
$$

where $T=G C G^{-1}$.

Received October 7, 1985.

1980 Mathematics Subject Classification (1985 Revision). Primary 65C05, 65C10.

Key words and phrases. $M$-sequence, GFSR algorithm, quasi-Monte Carlo methods, discrepancy, random numbers.

${ }^{*} x^{t}$ is the transpose of a vector or a matrix $x$. 
2. Discrepancy of GFSR Sequences. Recently, the $t$-dimensional discrepancy of GFSR sequences has been obtained in [3]. Let $r\left(L_{1}, L_{2}, \ldots, L_{t}\right)$ be the rank of the following set of row vectors,

$$
\left\{T^{i_{j}} \mid j=1,2, \ldots, L_{i} \text { for } i=1,2, \ldots, t\right\}
$$

where $T^{i j}$ is the $j$ th row vector of $T^{i}$ and $L_{i} \geq 0$ for $1 \leq i \leq t$. Let $r$ min be the minimum of $r\left(L_{1}, L_{2}, \ldots, L_{t}\right)$ such that $r\left(L_{1}, L_{2}, \ldots, L_{t}\right)$ is not full. Note that $r \min \leq p$. Then we have obtained the following theorem.

THEOREM D. The t-dimensional discrepancy of GFSR sequences with period $2^{p}-1$ is

$$
D_{N}^{(t)}=O\left((\log M)^{t} C \max \right),
$$

where $M=2^{p}, N=2^{p}-1$ and $C \max =2^{-r \min }$.

3. Optimal GFSR Generators in High Dimensions. We have defined the optimal generators for GFSR sequences in [3]. The definition is as follows.

Definition. When $r \min =p$, we call the GFSR generator 'optimal', where $p$ is the degree of the primitive polynomial.

Example. The following generator G1 is an optimal GFSR generator with $f(D)=$ $D^{7}+D^{4}+1$ :

$$
\mathrm{G} 1=\left[\begin{array}{lllllll}
1 & 0 & 0 & 0 & 0 & 0 & 0 \\
1 & 1 & 0 & 1 & 0 & 0 & 1 \\
0 & 0 & 0 & 1 & 1 & 0 & 1 \\
1 & 1 & 1 & 1 & 0 & 1 & 0 \\
1 & 1 & 1 & 1 & 1 & 1 & 1 \\
0 & 1 & 0 & 0 & 1 & 1 & 1 \\
1 & 1 & 0 & 0 & 1 & 1 & 0
\end{array}\right]
$$

The purpose of this paper is to prove the following theorem. Here we consider the case of $t(\leq p)$ dimensions.

THEOREM. In $t(\geq 4)$ dimensions, no optimal GFSR generators exist.

Proof. Consider the following linear equation,

$$
\sum_{i=1}^{t} \sum_{j=1}^{L_{i}} T^{i_{j}} w_{i j}=(0,0, \ldots, 0)
$$

where $w_{i j}$ is over $\mathrm{GF}(2)$.

Note that the above equation has a nonzero solution if and only if the $T^{i_{j}}$ 's are linearly dependent. The solution of $(3.1)$ is said to be in a class $\left(L_{1}, L_{2}, \ldots, L_{t}\right)$ if $w_{i, L_{i}}=1$ for all $1 \leq i \leq t$.

Denote by $C\left(L_{1}, L_{2}, \ldots, L_{t}\right)$ the number of nonzero solutions of (3.1) in a class $\left(L_{1}, L_{2}, \ldots, L_{t}\right)$. By using the principle of inclusion and exclusion, for $L_{i} \geq 1$, $i=1,2, \ldots, t$, we have

$$
C\left(L_{1}, L_{2}, \ldots, L_{t}\right)=\sum_{i=0}^{t}(-1)^{i} \sum_{0<j_{1}<j_{2}<\cdots<j_{i} \leq t} 2^{\sum_{k=1}^{t} L_{k}-i-\rho\left(j_{1}, j_{2}, \ldots, j_{i}\right)},
$$


where $\rho\left(j_{1}, j_{2}, \ldots, j_{i}\right)$ is equal to $r\left(f_{1}, f_{2}, \ldots, f_{t}\right)$ with

$$
\begin{aligned}
f_{n} & =L_{n}-1 & & \text { for } n=j_{1}, j_{2}, \ldots, j_{i}, \\
& =L_{n} & & \text { otherwise, }
\end{aligned}
$$

and where $0<j_{1}<j_{2}<\cdots<j_{i} \leq t$ and $n=1,2, \ldots, t$.

Assume that $r \min =p$ in $t$ dimensions. Consider a class $\left(L_{1}, L_{2}, \ldots, L_{t}\right)$ such that $\sum_{i=1}^{t} L_{i}=p+2$ with $L_{i} \geq 1$. Then

$$
\begin{aligned}
C\left(L_{1}, L_{2}, \ldots, L_{t}\right) & =2^{p+2-p}-{ }_{t} C_{1} 2^{p+1-p}+\sum_{i=2}^{t}(-1)^{i}{ }_{t} C_{i} \\
& =4-2 t+t-1=3-t \geq 0 .
\end{aligned}
$$

Therefore, $t$ must be smaller than 4 .

Tokyo Research Laboratory

IBM Japan, Ltd.

5-19 Sanbancho, Chiyoda-ku

Tokyo 102, Japan

1. M. Fushimi \& S. Tezuka, "The $k$-distribution of Generalized Feedback Shift Register pseudorandom numbers," Comm. ACM, v. 26, 1983, pp. 516-523.

2. T. G. LEWIS \& W. H. PAYNE, "Generalized Feedback Shift Register pseudorandom number algorithms," J. Assoc. Comput. Mach., v. 20, 1973, pp. 456-468.

3. S. TEZUKA, "On the discrepancy of GFSR pseudorandom numbers," J. Assoc. Comput. Mach., v. 34, 1987. 